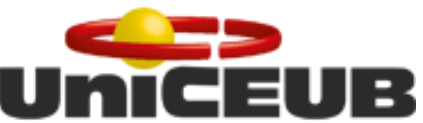 \\ Centro Unıversitárıo de Brasílıa \\ CENTRO UNIVERSITÁRIO DE BRASÍLIA - UniCEUB \\ PROGRAMA DE INICIAÇÃO CIENTÍFICA
}

LUCAS CARNEIRO NASCIMENTO PEREIRA

JONAS DE SOUZA FINCO

ESTUDO FARMACOECONÔMICO NO TRATAMENTO DE DOENÇA DE CROHN DE MODERADA A GRAVE

BRASÍLIA

2018 


\section{UnICEUB \\ Centro Unıversitárıo de Brasílıa}

\section{LUCAS CARNEIRO NASCIMENTO PEREIRA}

JONAS DE SOUZA FINCO

\section{ESTUDO FARMACOECONÔMICO NO TRATAMENTO DE DOENÇA DE CROHN DE}

MODERADA A GRAVE

Relatório final de pesquisa de Iniciação Científica apresentado à Assessoria de Pós-Graduação e Pesquisa.

Orientação: Dr. João Batista Monteiro Tajra.

\section{BRASÍLIA}

2018 
PIC VOLUNTÁRIO/UnICEUB

\title{
ESTUDO FARMACOECONÔMICO NO TRATAMENTO DE DOENÇA DE CROHN MODERADA A SEVERA
}

\author{
Lucas Carneiro Nascimento Pereira - UniCEUB, PIC Institucional, aluno voluntário \\ carneiroplucas@sempreceub.com
}

Jonas de Souza Finco - UniCEUB, PIC institucional, aluno voluntário

jonas.finco@sempreceub.com)

João Batista Monteiro Tajra - UniCEUB, professor orientador

jbtajra@uol.com.br

As Doenças Inflamatórios Intestinais são doenças crônicas caracterizadas por distúrbios gastrointestinais e extra-intestinais, sendo Doença de Crohn (DC) e Retocolite Ulcerativa (RCUI) as principais. A maior incidência, encontra-se entre vinte e quarenta anos de vida, impactando na capacidade laboral e qualidade de vida, refletindo prejuízos psicossociais e socioeconômicos para o paciente e para sistema de saúde. Avanços referentes a etiopatogenia dessas afecções permitiram melhora em seu tratamento, especialmente na DC. As terapias biológicas com agentes anti-TNF $\alpha$ isolados ou combinados são consideradas atualmente as mais efetivas formas de tratamento na indução e manutenção da remissão clínica em pacientes com DC moderada a severa, além de diminuir o risco de hospitalização e cirurgia. Portanto, o intuito do estudo foi analisar o custo direto e a efetividade do tratamento de pacientes com DC moderada a severa que utilizam a terapia imunossupressora, assim como definir seu perfil epidemiológico. Trata-se de um estudo observacional de coorte aberta, prospectivo com mínimo de duas consultas por paciente incluído no trabalho, durante período de seguimento de um ano após entrada, por inscrição online e voluntária ou marcação direta no ambulatório de coloproctologia do Hospital de Base do Distrito Federal. O diagnóstico de DC se realizou através de critérios clínicos, endoscópicos, radiográficos e histológicos e cada paciente foi classificado quanto o CDAI (Índice de Atividade da Doença de Crohn), sendo destinada a terapia biológica (Infliximabe ou Adalimumabe) àqueles com doença moderada a grave. Analisou-se o custo das drogas biológicas isoladas, não sendo somados os custos indiretos. Os preços de mercado foram obtidos através dos registros de compra de medicamentos pelo Banco de preços em Saúde pelo Ministério da Saúde e o desfecho clínico favorável como foi definido CDAI $<220$ ao final do período de observação, não representando doença ativa. Acompanhou-se 22 pacientes, 15 fizeram uso de terapia biológica (isolada ou combinada). Dessa forma o tratamento de manutenção do Adalimumabe (posologia de $40 \mathrm{mg}$ a cada duas semanas) representou custo médio anual por paciente de $\mathrm{R} \$ 61.032,00$, já o Infliximabe (posologia peso-dependente de $5 \mathrm{mg} / \mathrm{kg}$ a cada 8 semanas), considerando $65 \mathrm{~kg}$ o peso médio dos indivíduos com DC na 
Coorte, R\$ 31.663,77. Tal grupo da coorte apresentou média de 6,9 anos de doença, portanto, representando gasto considerável ao serviço de saúde pública nesse período de história natural da doença. O tempo médio de remissão clínica foi 6,75 meses para o infliximabe e 5,85 meses para o Adalimumabe. O perfil epidemiológico mostrou idade média de 36,1 anos, predomínio de mulheres (73\%), IMC médio de $24,4 \mathrm{Kg} / \mathrm{m}^{2}$, maioria étnica de pardos (50\%) e fenótipo de DC predominante íleo-colônico (diferindo da literatura). Cerca de 20,83\% necessitaram de cirurgia (40\% utilizaram terapia biológica após cirurgia como tratamento de manutenção). Neste estudo, o Infliximabe apresentou taxa 1,153 vezes maior e custo direto baseado no preço de mercado aproximadamente 3 vezes inferior ao Adalimumabe. À medida que novas drogas biológicas concorrentes continuam a chegar no mercado faz-se necessário o desenvolvimento de estudos centrados em análises farmacoeconômicas.

Palavras-Chave: Doença de Crohn. Anti TNF's. Biológicos. Doenças Inflamatórias Intestinais. 


\section{Sumário:}

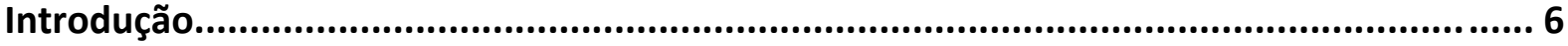

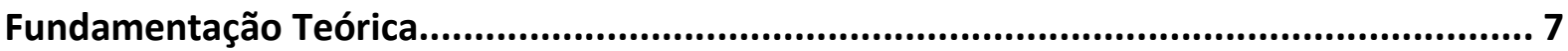

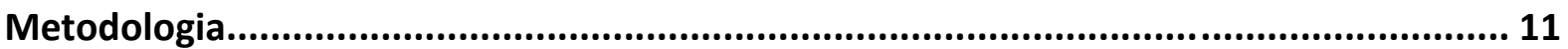

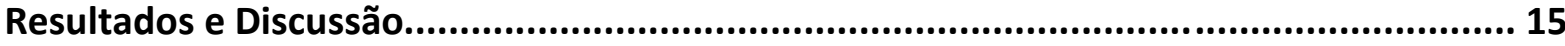

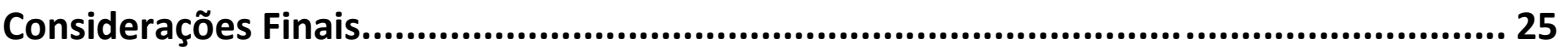

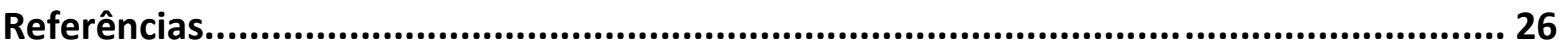




\section{INTRODUÇÃO:}

A presente pesquisa é um estudo quantitativo analítico observacional de estudo de Coorte prospectiva em que analisou o custo-efetividade do tratamento com terapia biológica Anti-TNF alfa para pacientes portadores de Doença de Crohn em hospital terciário do Distrito Federal durante período de um ano.

As Doenças Inflamatórios intestinais (DII) são doenças crônicas caracterizadas por distúrbios gastrointestinais e extra-intestinais, sendo Doença de Crohn (DC) e Retocolite Ulcerativa (RCUI) as principais enfermidades.

A incidência das DIl é mais evidente entre vinte e quarenta anos de vida ocasionando grande impacto na educação, capacidade de trabalho, produtividade a longo prazo, vida social e qualidade de vida dos pacientes. Dessa forma, as DII geram prejuízos psicossociais e, especialmente socioeconômicos, tanto para o próprio paciente como para sistema de saúde.(STARK; KÖNIG; LEIDL, 2006).

Estudo realizado no centro-oeste do São Paulo demonstra aumento progressivo da incidência de DC, com uma prevalência de 14,81/1.000 habitantes, condizente com taxas apresentadas com países da américa Latina e sul da Europa. (VICTORIA et al., 2009).

Além disso, em uma revisão sistemática realizada em 2017 analisou a mudança prevalência e incidência das doenças inflamatórias intestinais em diferentes países desde o ano de 1990, tanto para Doença de Crohn como a Retocolite Ulcerativa. (MOLODECKY et al., 2012). O estudo evidencia que a incidência das DII se mantivera estável na Europa, América do Norte, apesar de um significativo aumento da prevalência. Em contraste, países recémindustrializados como Ásia, África e América do Sul, incluindo o Brasil, apresentaram um aumento importante da incidência. O estudo sugere o recente processo de urbanização e industrialização vivenciada por estes países como principal justificativa desse aumento. Concluindo que os dados encontrados representam um desafio importante para sistemas de cuidado em saúde devido à complexidade e o custo relacionados a essas doenças. (SIEW et al., 2017).

Avanços referentes a etiopatogenia das afecções Inflamatórias Intestinais permitiram consequente grande avanço também em seu tratamento, especialmente em se tratando da Doença de Crohn. Estudos demonstraram que as terapias biológicas com agentes anti-fator de necrose tumoral- $\alpha$ isolado ou em combinação com imunomodulador são consideradas na 
atualidade as mais efetivas formas de tratamento na indução e manutenção da remissão clínica em pacientes com DC, além de diminuir o risco de hospitalização e cirurgia (SINGH et al., 2016).

Essa temática também tem sido discutida em inúmeros países como parte de uma política pública que serve de instrumento de auxílio na distribuição de recursos sanitários de uma forma mais justa e equilibrada, contribuindo para o uso racional do medicamento ao incorporar o custo aos quesitos de segurança, eficácia e qualidade das diferentes terapias medicamentosas (KHANNA; FEAGAN, 2017).

Têm-se relatado um aumento na tendência de uso destes fármacos para tratamento das enfermidades relacionadas a atividade inflamatória sistêmica, onde inclui-se a DC. Em um famoso estudo realizado na França, o custo da terapia Anti- TNF $\alpha$ foi estimado em $12.000,00$ euros por ano/paciente comparada a um custo de 360 euros por ano/paciente do uso de Azatioprina (PIERRE-OLIVIER et al., 2016).

Assim sendo, os dois tipos de estudos econômicos mais frequentes em DII, são o custo da avaliação da doença que quantifica o custo global das doenças e identificam fatores específicos de custo e o de comparações de custo-utilidade que avaliaram a relação custoeficácia relativa das alternativas de tratamento; Logo, técnicas de análise farmacoeconômica adequadas são de suma importância no auxílio aos responsáveis pela tomada de decisão sobre a alocação de recursos em cuidados de saúde no gerenciamento de DII (KHANNA; FEAGAN, 2017).

Portanto, o intuito do presente estudo foi analisar o custo direto e efetividade do tratamento de pacientes com DC moderada a severa que utilizam a terapia imunossupressora, assim como definir perfil epidemiológico da coorte estudada.

\section{FUNDAMENTAÇÃO TEÓRICA:}

As Doenças Inflamatórias Intestinais (DII) são doenças crônicas caracterizadas por desordens gastrointestinais e extra-intestinais e inclui dois tipos principais de enfermidade, a Doença de Crohn (DC) e a Retocolite Ulcerativa (RCUI). Uma revisão sistemática de 2012, em base de dados de 1950 a 2010 verificou um aumento na prevalência e incidência das Doenças Inflamatórias Intestinais, embora existam poucos dados epidemiológicos nos países em todo o mundo (MOLODECKY et al., 2012). 
Acredita-se existir associação das doenças inflamatórias intestinais com a industrialização das nações, visto estarem crescendo em incidência em países desenvolvidos a medida que se tornam industrializados, consequentemente esse grupo de patologias aparenta estar emergindo como uma afecção global; somado ao fato de universalmente suas taxas de incidência serem mais evidentes entre a segunda e quarta década de vida, afetando deste modo, a qualidade de vida de indivíduos em seus principais anos de vida com capacidade produtiva, ocasionando gastos a longo prazo para o paciente, para o sistema de saúde e para a sociedade em geral. (MOLODECKY et al., 2012)

A patogenia das doenças inflamatórias é complexa e inclui fatores genéticos de predisposição e fatores imunes de desequilíbrio desencadeados por antígenos de alimentos e bactérias do aparelho digestório. Os modelos de etiopatogenia das doenças inflamatórias sugerem três fatores essenciais no seu desenvolvimento: quebra na função de barreira intestinal, exposição das células imunes da lâmina própria intestinal aos antígenos intraluminais e uma resposta exagerada do sistema imune (CLAYBURGH; TURNER, 2004) . As evidências atuais indicam que o evento inicial no desenvolvimento seja o aumento da permeabilidade intestinal onde estão relatados: expressão alterada nas junções epiteliais intestinais (tight junctions -TJ) particularmente nas ocludinas e claudinas do tipo 3,4,5 e 8, aumento na apoptose epitelial e aumento nos níveis de TNF $\alpha$ e Interferon $\curlyvee$ principalmente nas mucosas intestinais (GROSCHWITZ, 2009).

Entre os fatores genéticos a susceptibilidade a DC foi mapeada no cromossoma 16, inicialmente por Hugot et al em 2001 com a descoberta do polimorfismo do gene NOD2/CARD15 (HUGOT, 2001). O gene NOD2 (nucleotide binding oligomerization domain containing gene 2), consiste de 12 éxons e 11 íntrons e é responsável pela codificação de uma proteína que atua como receptor proteico de células imunológicas principalmente monócitos, células dendríticas e enterócitos, onde atua como receptor bacteriano intracelular com ativação do fator Kappa $\beta$ (NF- $\kappa \beta)$, especificamente após o reconhecimento do componente bacteriano de parede muramyl dipeptídeo (MDP) (INOHARA, 2003). Mais de 40 mutações foram identificadas no gene NOD2, sendo as mais comuns a Arg702Trp no éxon 4 (substituição da arginina por triptofano na posição 702), Gly908Arg no éxon 8 (substituição da glicina por arginina na posição 908) e a inserção de carbono no éxon 11 (BOUKERCHA, 2015). 
O fator de necrose tumoral $\alpha$ (TNF $\alpha)$ é uma citocina pró-inflamatória sintetizada principalmente por fagócitos mononucleares, indutora de proliferação e diferenciação imune, sua via de sinalização regula a expressão gênica e moléculas de adesão (NIELSEN, 2013). Estudos iniciais in vitro mostraram que as células da imunidade de mucosa produziam níveis aumentados de TNF- $\alpha$ em pacientes com DC e que o processo de inflamação de mucosa destes pacientes reflete uma mudança no nível de citocinas produzido por células $T$ (TARGAN et al., 1997).

O primeiro agente Anti-TNFa aprovado na Europa para DC foi o Infliximab em 1999, sendo em 2006 também liberado para RCU nas formas de classificação moderada e grave das doenças (PIERRE-OLIVIER et al., 2016). Esta substância é um anticorpo quimérico (25\% murinho; $75 \%$ humano) que se liga especificamente ao TNF $\alpha$ com resultado direto no bloqueio do TNF $\alpha$ solúvel ou transmembrana e indireto pela indução de apoptose de linfócitos T (TRACEY et al., 2007). Por possuir esta constituição, o principal problema de sua administração é o desenvolvimento de anticorpos humanos contra o anticorpo quimérico (RUTGEERTS; VAN ASSCHE, 2009). A segurança e eficácia do Infliximab em DC foram avaliadas em dois ensaios clínicos, o estudo ACCENT I publicado em 2002, demonstrou que a terapia programada de infliximab com doses de $5 \mathrm{mg} / \mathrm{kg}$ e $10 \mathrm{mg} / \mathrm{kg}$ foram superiores ao placebo em DC com fístulas e de classificação moderada a grave (HANAUER et al., 2002).

O segundo agente aprovado para Doenças Inflamatórias foi o Adalimumab com o mecanismo de ação anti-TNF $\alpha$ e constituição recombinante humana monoclonal do tipo IgG1 ligando-se especificamente ao TNF, neutralizando sua função biológica através dos receptores p55 e p75 na superfície celular (RUTGEERTS; VAN ASSCHE, 2009).

Recentemente, em Janeiro de 2017 foi adicionado o Certolizumabe pegol para tratamento através da Portaria no 1, de 4 de janeiro de 2017, baseado no relatório de recomendação da Comissão Nacional de Incorporação de tecnologia do SUS (CONITEC) reportando que estudos científicos não demonstram diferença significativa entre certolizumabe pegol, adalimumabe e infliximabe no tratamento da Doença de Crohn em relação aos desfechos de indução, manutenção de remissão e resposta clínica. Essa conclusão foi alcançada pela comparação indireta dos resultados de uma revisão sistemática e meta-análise realizada por STIDHAM et al. (2014b) em que analisa 10 ensaios clínicos randomizados, dos quais dois avaliaram o infliximabe, quatro estudaram o adalimumabe e quatro tiverem o certolizumabe pegol como foco. Em cada umas das 10 pesquisas os 
medicamentos foram comparados com placebo. Além disso, estudos realizados demonstram uma redução nos custos diretos entre 40 a $50 \%$ do que os outros dois medicamentos no primeiro ano de tratamento. (Ministério da Saúde, 2017)

O tratamento das enfermidades, com frequência, era deficitário no objetivo de indução do estado de remissão do paciente acometido e também para manutenção do controle da doença ou sua cura, até a década de 90, época em que se inseriu o primeiro biológico no mercado e na clínica. Com o uso de corticosteroides, grande parcela dos tratados são resistentes a este método terapêutico desde o início ou findam em corticoidedependência, apresentando eficácia limitada e efeitos colaterais consideráveis; o uso de 5 ASA (ácido 5-aminosalicílico), de imunossupressores e imunorreguladores também apresentam o viés de expor o paciente ao risco de infecções oportunistas. Sendo os anticorpos monoclonais, como Inflixumab, Adalimumab e Certolizumabe, as opções mais recentes e promissoras. (SINGH et al., 2016)

Em diversos países está relatado um aumento na tendência de uso destes fármacos para tratamento das enfermidades relacionadas a atividade inflamatória sistêmicas, incluindo DC e RCU. Em um estudo na França, o custo da terapia Anti- TNFa foi estimado em $12.000,00$ euros por ano/paciente comparada a um custo de 360 euros por ano/paciente do uso de Azatioprina (PIERRE-OLIVIER et al., 2016).

Em geral, a DC acomete indivíduos jovens/adultos jovens e por não apresentar um tratamento curativo requerem uma terapia a longo prazo gerando um aumento do custo devido a provisão de cuidados e perda de produtividade econômica. Além do fato de que o tratamento lida com o sistema imunológico e suas respostas, ocorrendo efeitos adversos relacionados e infecções específicas consequentes, onde com frequência os pacientes requerem hospitalizações ou manejos cirúrgicos, que também aumentam o custo da terapêutica. Logo, sendo a sobrecarga econômica devidamente alta, tanto para o paciente quanto para o sistema de saúde. (KHANNA; FEAGAN, 2017)

No Brasil com a publicação da portaria 1.318 de 2002, com inclusão recente do Certolizumab em 2017, os pacientes brasileiros diagnosticados com DC ou RCU podem obter medicamentos para o tratamento das suas doenças pelo Sistema Único de Saúde (SUS) sendo no total sete destinados para as doenças inflamatórias intestinais, incluindo os AntiTNFs Infliximab, Adalimumab e atualmente Certolizumab. Os custos desta terapia e as 
tendências de uso destes fármacos no Brasil não possuem estudos comparativos relacionados a farmacoeconomia.

\section{METODOLOGIA:}

O estudo foi aprovado pelo Comitê de Ética e Pesquisa do Centro Universitário de Brasília (UniCEUB) com número do parecer 2.514.196 e CAAE: 79111716.0.0000.5553.

Trata-se de um estudo quantitativo analítico observacional de estudo de Coorte do tipo aberta unicêntrica, prospectivo com seguimento mínimo de duas consultas por paciente incluído no espaço amostral durante período de um ano após a entrada realizada no ambulatório de coloproctologia do Hospital de Base do Distrito Federal entre agosto de 2017 e fevereiro de 2018.

A inscrição para a coorte foi estabelecida de forma online e voluntária através do endereço eletrônico www.hospitaldebasedf.com.br e por marcação direta no ambulatório acima citado. Durante o período de estudo de um ano foram inscritos 171 pacientes, os quais foram admitidos como parte integrante da coorte somente os que cumpriram os seguintes critérios de inclusão e de exclusão:

a) Critérios de Inclusão:

1- Diagnóstico de DC com doença de intestino delgado ou cólon como local primário.

2-Pacientes entre 18 e 70 anos, de ambos os sexos.

3- Indicação ou em uso de terapia Imunossupressora.

b) Critérios de Exclusão:

1- Pacientes Grávidas ou em lactação.

2- Pacientes Menores de 18 anos.

3- Presença de Malignidade

4- Pacientes com nefropatia.

5- Pacientes com Hepatopatia comprovada ou suspeita. (TGO/TGP $>2 x$ ).

6 - Paciente em uso de corticoterapia. 
Dos 171 indivíduos inscritos, 95 tornaram-se elegíveis com Doença Inflamatória Intestinal, sendo 54 diagnosticados com Retocolite Ulcerativa. Portanto, 41 pacientes admitidos na Coorte com Doença de Crohn.

Os pacientes incluídos no estudo foram confirmados os diagnósticos de Doença de Crohn através de critérios clínicos, endoscópicos, radiográficos e histológicos padrões. Durante período de um ano, pacientes foram acompanhados por pelo menos duas consultas, segundo critério médico especializado. Em todas as consultas foram mensurados IMC, CDAI (Crohn's Disease Activity Index), classificação de Montreal, tipo de tratamento e presença/ausência de manifestações extraintestinais.

Inicialmente, os pacientes foram classificados baseados nos Critérios de Montreal) e no Índice de Atividade da Doença de Crohn (CDAl, do inglês Crohn's Disease Activity Index). A Classificação de Montreal permite avaliação fenotípica com critérios clínicos, a qual inclui idade do diagnóstico, comportamento e localização da doença de Crohn proposta em 2005 (SILVERBERG et al., 2005)(REBELO et al., 2011), detalhada na Tabela 1. Já o CDAl é o método considerado padrão-ouro para a caracterização dos estágios de tal doença (SANDBORN et al.; 2002), detalhado na Tabela 2. O índice tem valores padronizados para definição da atividade da doença, sendo considerada em remissão quando o CDAl é inferior a 150; leve a moderada quando o CDAl oscila entre 150 e 219; moderada a grave entre 220 e 450; e grave ou fulminante quando os valores são superiores a 450.

Tabela 1. Classificação de Montreal para Doença de Crohn (SILVERBERG et al., 2005) (REBELO et al., 2011)

\begin{tabular}{|c|c|c|}
\hline Idade do Diagnóstico & & Modificadores \\
\hline A1 & $\leq 16$ anos & \\
\hline A2 & $17-40$ anos & \\
\hline A3 & $\geq 40$ anos & \\
\hline Localização & & \\
\hline L1 & Íleo terminal & $\mathrm{L} 1+\mathrm{L} 4^{*}$ \\
\hline L2 & Cólon & $\mathrm{L} 2+\mathrm{L} 4^{*}$ \\
\hline L3 & Íleo-cólon & $\mathrm{L} 3+\mathrm{L} 4^{*}$ \\
\hline L4 & Trato gastrointestinal & \\
\hline
\end{tabular}




\begin{tabular}{|c|c|c|}
\hline & superior & \\
\hline Comportamento & & \\
\hline B1 & $\begin{array}{c}\text { Não estenosante/não } \\
\text { penetrante }\end{array}$ & B1p** \\
\hline B2 & Estenosante & B2p** \\
\hline B3 & Penetrante & B3p** \\
\hline
\end{tabular}

* O modificador trato gastrointestinal superior (L4) permite a co-classificação da localização L4 com L1 a L3

** Modificador doença perianal ( $p)$, acrescentado a B1-B3, se doença perianal concomitante $\S$ A categoria B1 deve ser considerada provisória até ter decorrido um período pré-definido de tempo desde o diagnóstico da doença (geralmente 5 anos).

Tabela 2. Cálculo do Índice de Atividade da Doença de Crohn (Crohn's Disease Activity Index adaptado de BEST et al. ).

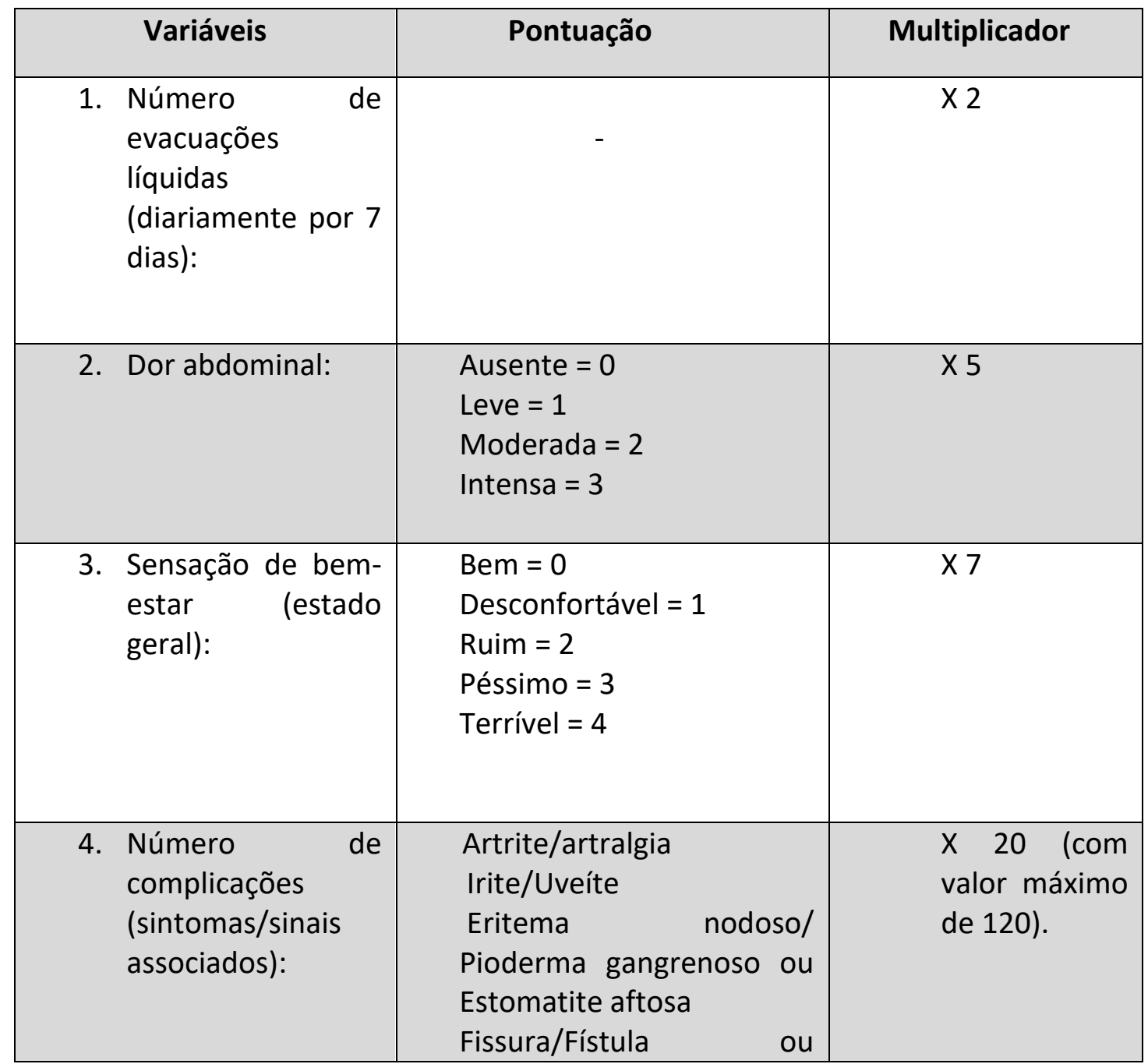




\begin{tabular}{|c|c|c|}
\hline & $\begin{array}{l}\text { Abscesso anal } \\
\text { Outras fístulas } \\
\text { Febre }>37,80 \mathrm{C}\end{array}$ & \\
\hline $\begin{array}{lll}\text { 5. Uso } & \text { de } \\
\text { antidiarreicos } & \\
\text { (difelonato } & \text { ou } \\
\text { loperamida): } & \end{array}$ & $\begin{array}{l}\text { Não }=0 \\
\text { Sim }=1\end{array}$ & $\times 30$ \\
\hline 6. Massa abdominal: & $\begin{array}{l}\text { Ausente }=0 \\
\text { Questionável }=1 \\
\text { Bem definida }=5\end{array}$ & $\mathrm{X} 10$ \\
\hline 7. Hematócrito & $\begin{array}{l}\text { Homens: } 47-\mathrm{Ht} \% \\
\text { Mulheres: } 42-\mathrm{Ht} \% \\
\text { (diminuir em vez de } \\
\text { somar, caso Ht do } \\
\text { paciente seja maior que o } \\
\text { padrão). }\end{array}$ & $\times 6$ \\
\hline 8. Peso: & $\begin{array}{l}1 \text { - peso / peso padrão } x \\
100 \\
\text { Adicione ou subtraia } \\
\text { segundo o sinal }\end{array}$ & $\mathrm{X} 1$ \\
\hline
\end{tabular}

Após essa primeira etapa de classificação dos pacientes, a prescrição de terapia biológica em pacientes com Doença de Crohn foi reservada aqueles que foram classificados com patologia mensurada de moderada a grave (com CDAl >220) sem resposta clínica significativa, após 6 semanas, à corticosteroides, azatioprina e metotrexato, ou com contraindicação ou intolerância a corticosteroides e imunossupressores ou ainda que tenha aderido ao estudo já em uso prévio da terapia.

O esquema terapêutico implementado no combate à doença engloba uma terapia inicial de indução e uma terapia subsequente de manutenção:

A posologia da terapia de indução com os anticorpos monoclonais Anti-fator de necrose tumoral (anti-TNF) seguiu as principais diretrizes nacionais e internacionais de uso demonstrada na Tabela 3. Sendo prescrito Infliximabe ( $5 \mathrm{mg} / \mathrm{kg}$ nas semanas 0,2 e 6 ) ou Adalimumabe (160 mg na semana 0 e $80 \mathrm{mg}$ na semana 2). Já a terapia de manutenção poderá ser continuada $(5 \mathrm{mg} / \mathrm{kg}$ de Infliximabe a cada 8 semanas ou $40 \mathrm{mg}$ de Adalimumabe a cada 2 semanas). 
Tabela 3. Esquemas de indução da remissão e manutenção de remissão das duas drogas.

\begin{tabular}{|l|c|c|}
\hline & Indução da remissão & Manutenção (1 ano) \\
\hline Infliximabe & $5 \mathrm{mg} / \mathrm{kg}$ de peso nas semanas 0, 2, 6 & $5 \mathrm{mg} / \mathrm{kg}$ de peso a cada 8 \\
& & semanas \\
\hline Adalimumabe & $160 \mathrm{mg}$ na semana 0 e $80 \mathrm{mg}$ na semana 2 & $40 \mathrm{mg}$ a cada 2 semanas \\
\hline
\end{tabular}

\section{Para análise do custo-efetividade:}

Segundo 3을 Consenso Europeu (3rd European Evidence-based Consensus on the Diagnosis and Management of Crohn's Disease 2016) foi definido que pacientes com CDAI >220 apresentam Doença de Crohn ativa; pacientes com redução igual ou superior a 100 pontos, após início da terapêutica, como apresentadores de resposta clínica significativa a terapia Anti-TNF's e ainda pacientes com CDAl < 150 considerados em remissão. Sendo dessa forma, caracterizada a efetividade da terapia de indução e/ou terapia de manutenção no objetivo de controle da doença. (GOMOLLÓN et al., 2017).

Foram analisados os preços de mercado das drogas biológicas isoladas, não sendo somados os custos indiretos e custos com imunossupressores, infusões ou outras drogas utilizadas em conjunto para o tratamento da DC. Os dados de custo direto baseado no preço de mercado para análise de custo-efetividade foram obtidos a partir de dados fornecidos pelo Banco de Preços em Saúde, disponibilizado pelo Ministério da Saúde - BPS, disponível < http://bps.saude.gov.br/visao/consultaPublica/relatorios/geral/index.jsf> acessados em junho de 2018. Os demais custos de tratamento foram obtidos de registros administrativos de gestão de custo de um hospital terciário do Distrito Federal.

\section{RESULTADOS E DISCUSSÃO:}

Baseado na inscrição inicial descrita em metodologia 171 indíviduos foram registrados. Desse universo, 95 foram elegíveis com Doença Inflamatória Intestinal (DII), sendo diagnosticada Retocolite Ulcerativa em 54 destes pacientes. No final, 41 pacientes (24\% do $\mathrm{n}$ total inicial) foram incluídos com Doença da Crohn (Gráfico 1). O perfil 
epidemiológico do espaço amostral de pacientes com Doença de Crohn utilizado no estudo está demonstrado de maneira detalhada na tabela 4.

Gráfico 1: "N" total de 171 pacientes, pormenorizando probabilística da população de estudo.

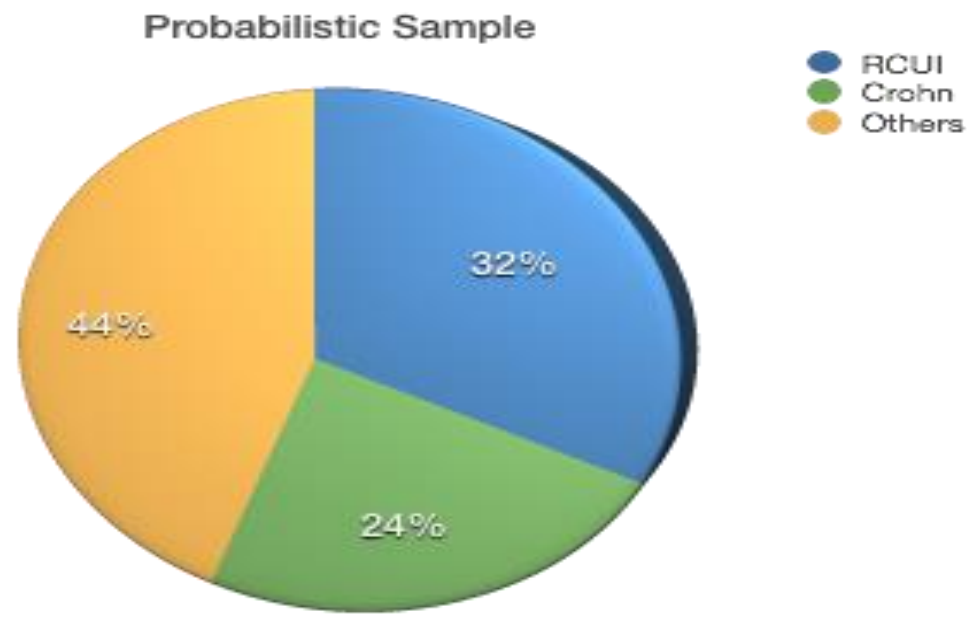

Tabela 4: Características Clínicas e Perfil Epidemiológico da Doença de Crohn ( $\mathrm{n}=41$ ).

\begin{tabular}{|c|c|}
\hline Características & Linha de Base \\
\hline Idade (anos) & $36,1 \pm 10$ \\
\hline $\begin{array}{l}\text { Sexo\%: } \\
\text { - Homem } \\
\text { - Mulher }\end{array}$ & $\begin{array}{l}27 \% \\
73 \%\end{array}$ \\
\hline Índice de Massa Corporal (IMC Kg/m²) & $24,4 \pm 3$ \\
\hline $\begin{array}{l}\text { Etnia \%: } \\
\qquad \begin{array}{l}\text { - } \\
\text { - } \\
\text { - } \\
\text { Pranco Legros americanos }\end{array}\end{array}$ & $\begin{array}{l}42 \% \\
50 \% \\
8 \%\end{array}$ \\
\hline Tempo de doença (em anos) & $6,9 \pm 5$ \\
\hline $\begin{array}{l}\text { Doença ativa com Índice de atividade da } \\
\text { Doença de Crohn }>220(\text { CDAl }>220)\end{array}$ & $30 \%$ \\
\hline
\end{tabular}




\begin{tabular}{|c|l|}
\hline Classificação fenótipo de Montreal: & \\
$\bullet \quad$ L1 & $4 \%$ \\
- L2 & $31 \%$ \\
- L3 & $54 \%$ \\
& $11 \%$ \\
\hline Tratamento Cirúrgico & $23 \%$ \\
\hline Tratamento Anti-TNFs & $41,6 \%$ \\
\hline Manifestações Extra-intestinais & $61 \%$ \\
\hline
\end{tabular}

No Brasil e América Latina, estudos epidemiológicos e relacionados às DII em geral, e especificamente a DC, ainda são escassos. Marcada por tradicionalmente ser uma doença de nações geograficamente localizadas no hemisfério norte, tal padrão epidemiológico vem sofrendo alterações significativas, com estudos sugerindo um incremento de casos na América do Sul, Ásia e África. Além disso, tal alteração na incidência dentre as distintas etnias e nacionalidades é fator de importância na compreensão da patogênese e influência de fatores ambientais na gênese da doença. Neste estudo, os dados estão de acordo com a literatura nacional e internacional, sendo RCUI mais prevalente que DC neste período de acompanhamento, acometendo predominantemente mulheres jovens. (NIELSEN, 2013).

O comportamento mais comum da doença no diagnóstico é do tipo inflamatório representando 65\% da amostra. A classificação de Montreal (2005) mostrou muitas diferenças em números de estudos de coorte em todo o mundo. Em um registro francês de base populacional, a doença colônica (L2) ocorreu em 40\% (TARGAN et al., 1997). Da mesma forma, em um estudo da Dinamarca, a prevalência de doença colônica foi de 37\% (PierreOlivier et al., 2016). Nossos dados mostraram um diferente fenótipo com grande predominância de Montreal L3 (54\%). Perante as várias classificações propostas para a DC, a de Montreal, introduzida em 2005, é a mais utilizada e tem como objetivo, uniformizar a caracterização da doença, utilizando parâmetros clínicos reprodutíveis e características epidemiológicas para que se possa correlacionar determinado fenótipo da doença com possíveis desfechos clínicos e com prognóstico, para que se selecione qual a melhor estratégia terapêutica e o seguimento mais adequado para cada paciente (BECHARA et al., 2015). 
Em um estudo de coorte de Copenhague, o grau de atividade clínica foi coletado prospectivamente. No primeiro ano após o diagnóstico, $80 \%$ apresentavam doença de alta atividade (TRACEY et al., 2007). Em nossa coorte, $30 \%$ dos pacientes estavam com doença de atividade clínica por CDAI. É possível que na nova era biológica esse número permaneça estável.

O tratamento cirúrgico em três coortes da Noruega, Hungria e Dinamarca relataram que a probabilidade de ressecção cirúrgica foi de 15\%, 30\% e 38 a 52\% em 1,5 e 10 anos após o diagnóstico (12), (13), (14). Em Brasília, 23\% dos pacientes foram submetidos ao tratamento cirúrgico, sendo $11 \%$ como primeiro tratamento uma sala de emergência com ileocolectomia direita.

O algoritmo de manejo convencional da DC baseia-se na etapa de atendimento sequencial, iniciando com os agentes corticosteroides, imunossupressores e no caso de doença de moderada a grave Anti-TNFs alfa (TAKESHIMA et al., 2016). Observamos, em contraste, uma preferência por antagonistas do TNF (Adalimumabe e Infliximabe) com 41,6\% como droga de escolha terapêutica. Possivelmente muitos clínicos abandonaram o algoritmo da pirâmide para uma abordagem que apresenta a introdução precoce de terapia altamente eficaz em pacientes de alto risco. O custo dessa escolha também foi objetivo de análise do presente estudo.

Manifestações extraintestinais das DII são observadas em $25-40 \%$ dos pacientes com RCUI e DC (LEE et al., 2017). Em um ano de acompanhamento, encontramos $61 \%$ de manifestações extraintestinais.

Dos 41 pacientes diagnosticado com DC, acompanhou-se 22 pacientes $(53,65 \%$ da amostra) em uso de terapia imunossupressora no ambulatório de DC no Hospital de Base do Distrito Federal com um mínimo de 2 consultas com cálculo do CDAl em cada uma delas. Estando o paciente em uso de terapia imunossupressora (seja Azatioprina AZA, Adalimumabe ADA ou Infliximabe INF, excetuando-se o uso de corticoterapia), compondo a coorte do estudo em questão. Destes, 7 pacientes fazem uso de Azatioprina e 15 pacientes utilizam terapia biológica (de forma isolada ou associada a AZA).

Do grupo que utilizou terapia biológica: 7 pacientes fizeram uso de ADA de forma pura, 4 pacientes utilizaram INF de forma pura, 1 paciente utilizou a combinação AZA + ADA e 3 pacientes trataram-se com combinação INF + AZA. Conforme fluxograma 1 abaixo: 
Fluxograma 1: Subdivisões da coorte de pacientes com DC, de acordo com terapêutica utilizada.

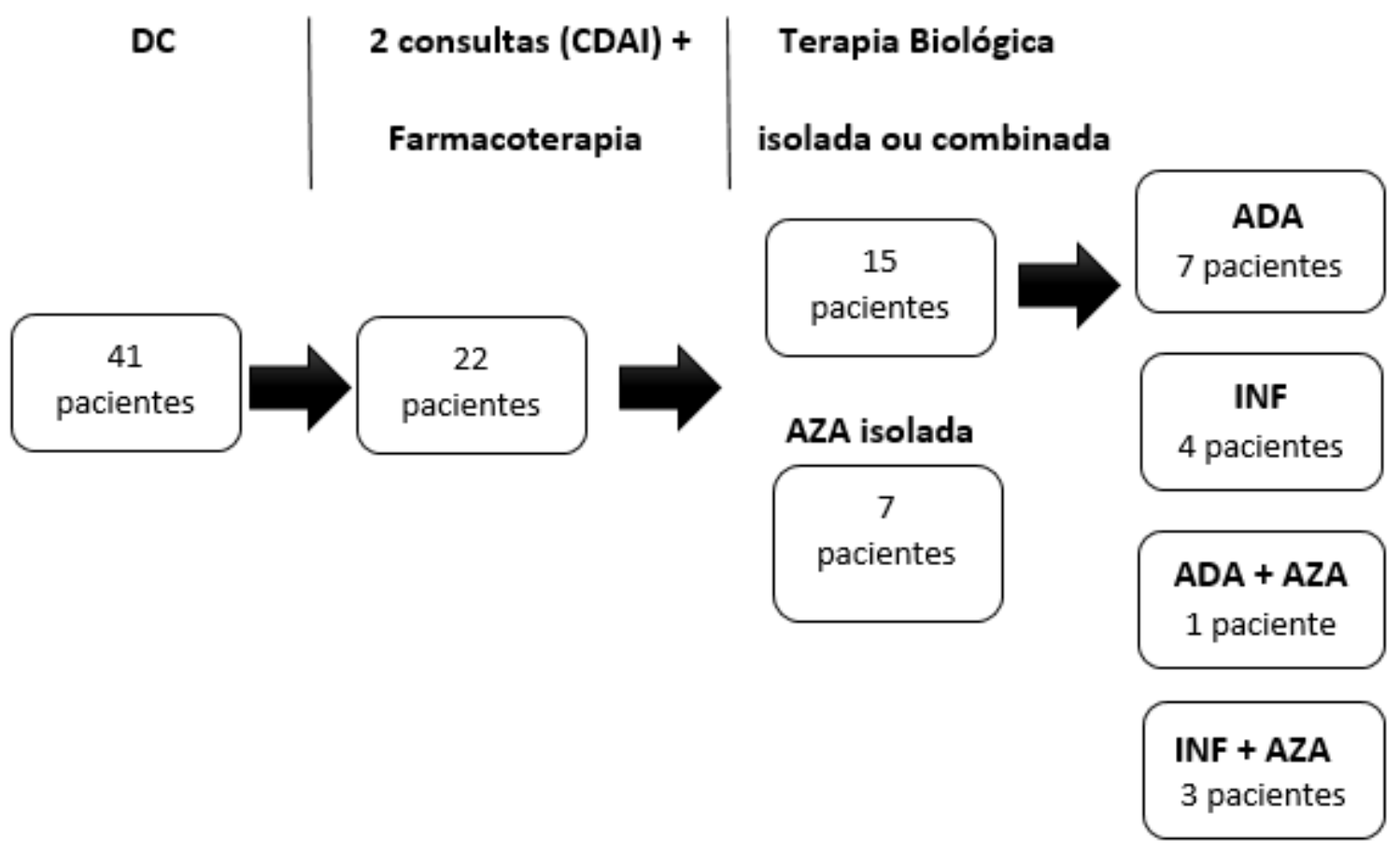

Baseado nesses dados, foi realizado uma projeção do custo de tratamento (de manutenção) anual médio dos pacientes em uso de terapia medicamentosa (AZA, ADA, INF), considerando suas respectivas posologias, onde nas que se configuram como pesodependente, utilizou-se a média de peso (em quilogramas) da coorte de pacientes: $65 \mathrm{~kg}$. Os preços de mercado utilizando o custo direto dos medicamentos foram obtidos a partir de dados fornecidos pelo Banco de Preços em Saúde, disponibilizado pelo Ministério da Saúde BPS, disponível < http://bps.saude.gov.br/visao/consultaPublica/relatorios/geral/index.jsf> acessados em junho de 2018 em que o preço unitário do Adalimumabe 40 mg foi de $\mathrm{R} \$$ 2.543,00; Infliximabe $100 \mathrm{mg}(10 \mathrm{mg} / \mathrm{ml})$ foi de $\mathrm{R} \$ 1.623,78$ e Azatioprina $50 \mathrm{mg}$ por $\mathrm{R} \$ 2,85$. 
Desta forma, considerando o mês com 4 semanas (consequentemente o período em análise - 1 ano - com 48 semanas) e as respectivas posologias de INF $5 \mathrm{mg} / \mathrm{kg}$ a cada 8

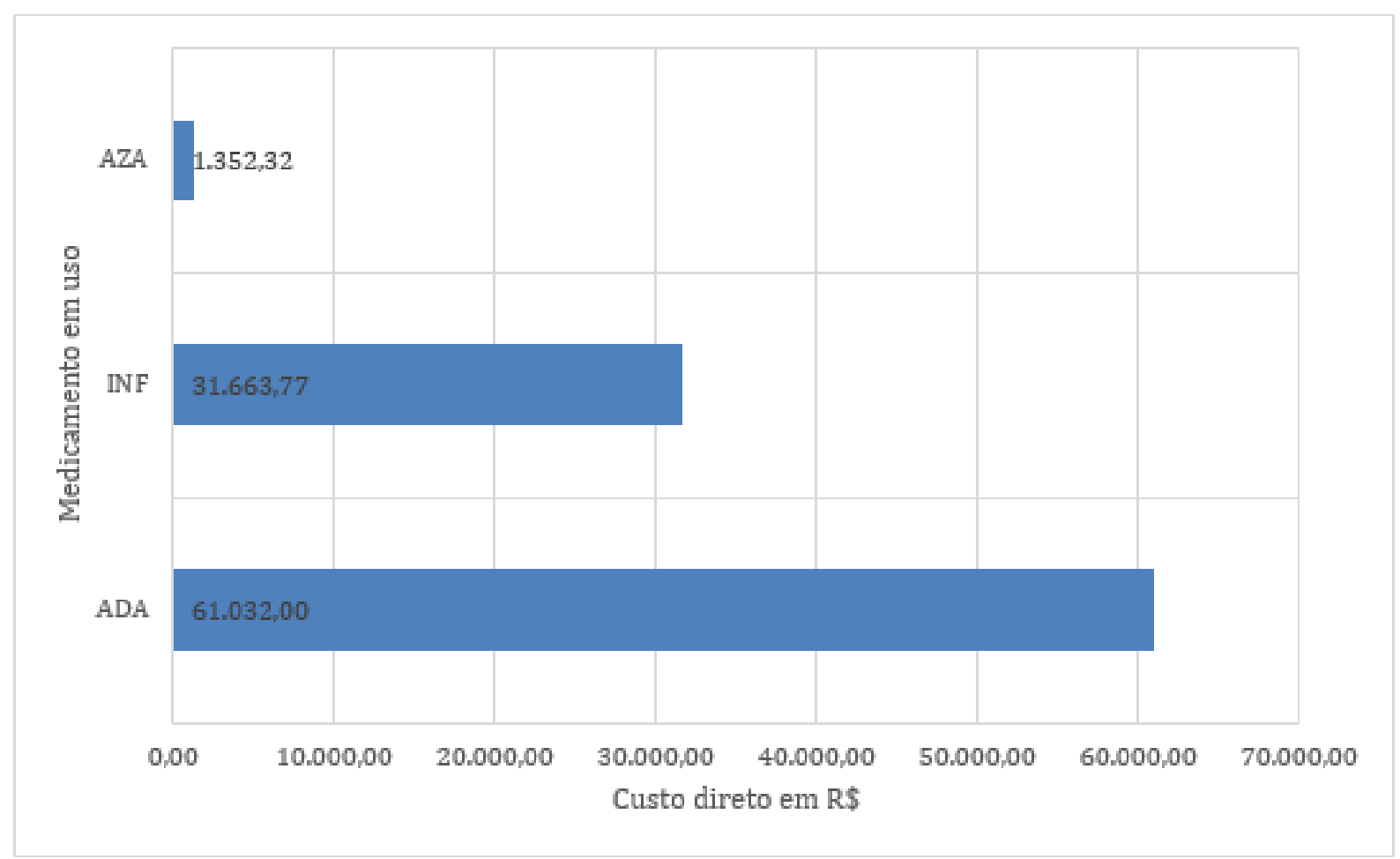

semanas, ADA $40 \mathrm{mg}$ a cada 2 semanas e AZA $1 \mathrm{mg} / \mathrm{kg} / \mathrm{dia}$ (de 50 a $150 \mathrm{mg} / \mathrm{dia}$ ), obteve-se o Gráfico 2: Projeção de custo médio do tratamento de manutenção anual por paciente diagnosticado com $D C . A D A=$ Adalimumabe, $I N F=$ infliximabe, $A Z A=$ azatioprina, $R \$=$ reais .

Além disso, é essencial analisar a relação projetada de custo anual com a média de tempo de doença (em anos) desses pacientes, o que representaria o impacto financeiro do tratamento com tais terapias: os 41 pacientes do grupo inicial apresentaram uma média de 6,9 anos de doença, representa-se o gasto de $R \$ 218.480,01$ (INF), R\$421.120,80 (ADA) e R\$ 9.331,08 (AZA) ao longo do tempo, com impacto no Sistema Único de Saúde (SUS) no Distrito Federal.

Dentre os 22 pacientes do estudo, obteve-se a tabela 5.

Tabela 5. Tabela descritiva da coorte em estudo com análise individualizada dos pacientes baseada nas seguintes característica: tratamento de manutenção utilizado, CDAI calculado em duas consultas distintas com intervalo mínimo de dois meses, tempo de terapia durante as consultas, custo direto total do tratamento, peso (em $\mathrm{kg}$ ) do paciente. 


\begin{tabular}{|c|c|c|c|c|c|c|}
\hline PACIENTE & TERAPIA & $\begin{array}{c}\text { CDAI } \\
\text { ENTRADA }\end{array}$ & $\begin{array}{c}\text { CDAI } \\
\text { CONSULTA }\end{array}$ & $\begin{array}{l}\text { TEMPO DE } \\
\text { REMISSÃO }\end{array}$ & $\begin{array}{c}\text { CUSTO } \\
\text { (R\$) }\end{array}$ & $\begin{array}{l}\text { PESO } \\
\text { (KG) }\end{array}$ \\
\hline PACIENTE 1 & ADA & 44 & 90 & $\begin{array}{l}6 \text { MESES } \\
\text { MANTIDA }\end{array}$ & $\begin{array}{c}R \$ \\
30.516\end{array}$ & 70,1 \\
\hline PACIENTE 2 & ADA & 389 & 474 & $\begin{array}{c}2 \text { MESES } \\
\text { ATIVA }\end{array}$ & $\begin{array}{c}R \$ \\
10.172\end{array}$ & 81,9 \\
\hline PACIENTE 3 & AZA & 218 & 61 & $\begin{array}{c}7 \text { MESES } \\
\text { REMISSÃO }\end{array}$ & $\begin{array}{c}\mathrm{R} \$ \\
837,9\end{array}$ & 70 \\
\hline PACIENTE 4 & AZA & 156 & 40 & $\begin{array}{l}10 \text { MESES } \\
\text { MANTIDA }\end{array}$ & $\begin{array}{c}\mathrm{R} \$ \\
1.453,5\end{array}$ & 85 \\
\hline PACIENTE 5 & $\begin{array}{c}\text { INF } \\
+ \\
\text { AZA }\end{array}$ & 180 & 269 & $\begin{array}{l}4 \text { MESES } \\
\text { ATIVA }\end{array}$ & $\begin{array}{c}R \$ \\
10.491 \\
5\end{array}$ & 62 \\
\hline PACIENTE 6 & $\begin{array}{c}\text { ADA } \\
+ \\
\text { AZA }\end{array}$ & 439 & 175 & $\begin{array}{c}4 \text { MESES } \\
\text { REMISSÃO }\end{array}$ & $\begin{array}{c}\text { R\$ } \\
20.747 \\
5\end{array}$ & 59 \\
\hline PACIENTE 7 & ADA & 48 & 28 & $\begin{array}{l}3 \text { MESES } \\
\text { MANTIDA }\end{array}$ & $\begin{array}{c}R \$ \\
15.258\end{array}$ & 63 \\
\hline PACIENTE 8 & INF & 391 & 299 & $\begin{array}{l}4 \text { MESES } \\
\text { ATIVA }\end{array}$ & $\begin{array}{c}\mathrm{R} \$ \\
8.329,9\end{array}$ & 51,3 \\
\hline PACIENTE 9 & AZA & 133 & 159 & $\begin{array}{l}3 \text { MESES } \\
\text { MANTIDA }\end{array}$ & $\begin{array}{c}R \$ \\
438,6\end{array}$ & 85,5 \\
\hline PACIENTE 10 & $\begin{array}{c}\text { INF } \\
+ \\
\text { AZA }\end{array}$ & 80 & 90 & $\begin{array}{l}6 \text { MESES } \\
\text { MANTIDA }\end{array}$ & $\begin{array}{c}R \$ \\
13.097, \\
4\end{array}$ & 51,6 \\
\hline PACIENTE 11 & AZA & 267 & 252 & $\begin{array}{c}7 \text { MESES } \\
\text { ATIVA }\end{array}$ & $\begin{array}{c}\mathrm{R} \$ \\
718,2\end{array}$ & 60 \\
\hline PACIENTE 12 & AZA & 61 & 50 & $\begin{array}{l}7 \text { MESES } \\
\text { MANTIDA }\end{array}$ & $\begin{array}{c}R \$ \\
682,29\end{array}$ & 57 \\
\hline PACIENTE 13 & $\begin{array}{c}\text { INF } \\
+ \\
\text { AZA }\end{array}$ & 309 & 43 & $\begin{array}{c}3 \text { MESES } \\
\text { REMISSÃO }\end{array}$ & $\begin{array}{c}\text { R\$ } \\
9.048,9\end{array}$ & 71,3 \\
\hline PACIENTE 14 & ADA & 214 & 35 & $\begin{array}{l}2 \text { MESES } \\
\text { MANTIDA }\end{array}$ & $\begin{array}{c}\text { R\$ } \\
10.172\end{array}$ & 66 \\
\hline PACIENTE 15 & INF & 197 & 58 & $\begin{array}{l}10 \text { MESES } \\
\text { MANTIDA }\end{array}$ & $\begin{array}{c}R \$ \\
20.703\end{array}$ & 51 \\
\hline
\end{tabular}




\begin{tabular}{|c|c|c|c|c|c|c|}
\hline & & & & & 1 & \\
\hline PACIENTE 16 & INF & 117 & 87 & $\begin{array}{l}8 \text { MESES } \\
\text { MANTIDA }\end{array}$ & $\begin{array}{c}\mathrm{RS} \\
25.980, \\
48\end{array}$ & 80 \\
\hline PACIENTE 17 & ADA & 53 & 171 & $\begin{array}{l}13 \text { MESES } \\
\text { MANTIDA }\end{array}$ & $\begin{array}{c}\mathrm{R} \$ \\
66.118\end{array}$ & 85 \\
\hline PACIENTE 18 & AZA & 173 & 56 & $\begin{array}{l}4 \text { MESES } \\
\text { MANTIDA }\end{array}$ & $\begin{array}{c}\mathrm{R} \$ \\
403,56\end{array}$ & 59 \\
\hline PACIENTE 19 & ADA & 205 & 131 & $\begin{array}{l}7 \text { meses } \\
\text { MANTIDA }\end{array}$ & $\begin{array}{c}R \dot{R} \\
35.602\end{array}$ & 62,5 \\
\hline Paciente 20 & AZA & 286 & 192 & $\begin{array}{c}7 \text { meses } \\
\text { REMISSÃO }\end{array}$ & $\begin{array}{c}\mathrm{R} \$ \\
927,6\end{array}$ & 77,5 \\
\hline Paciente 21 & INF & 222 & 256 & $\begin{array}{l}3 \text { MESES } \\
\text { ATIVA }\end{array}$ & $\begin{array}{c}R \$ \\
6.941,6 \\
5\end{array}$ & 57 \\
\hline PACIENTE 22 & ADA & 228 & 186 & $\begin{array}{c}6 \text { meses } \\
\text { REMISSÃo }\end{array}$ & $\begin{array}{c}\mathrm{R} \$ \\
30.516\end{array}$ & 73 \\
\hline
\end{tabular}

Foi utilizado o Consenso Europeu baseado em Evidência no Diagnóstico e Manejo da Doença de Crohn (2017) em que episódios agudos da doença são considerados como apresentando CDAl > 220, ponto escolhido como corte para um desfecho positivo/negativo no trabalho. Sendo assim, 8 pacientes (36,36\%) apresentaram-se já na primeira consulta (consulta de entrada) em episódio agudo e 5 pacientes $(22,72 \%)$ na consulta subsequente.

Relacionou-se as variáveis custo e desfecho clínico. O desfecho clínico foi analisado com base no CDAI realizado na segunda consulta. Pacientes que apresentaram manutenção do CDAl após início do tratamento (iniciando com CDAl $<220$ e assim mantendo-o) foram referidos como "mantidos"; Pacientes que apresentaram redução para níveis inferiores a 220 no CDAl (que inicialmente estavam com CDAl > 220, em episódio agudo), foram referidos como "desfecho clínico positivo", em remissão; Pacientes que apresentaram CDAI $>220$ na segunda consulta, indicando doença clinicamente ativa, foram nomeados como "ativos". Logo, divididos em 3 grupos (doença ativa + mantida + desfecho positivo), com os respectivos custos totais direto, evidenciado no gráfico 3.

Gráfico 3: Custo direto do tratamento de manutenção para alcançar desfecho clínico com base no CDAl. Azul: 12 pacientes "mantidos", com seu respectivo custo; Vermelho: 5 
pacientes com desfecho positivo, em remissão, com seu respectivo custo; Verde: 5 pacientes com doença clinicamente ativa, com seu respectivo custo.

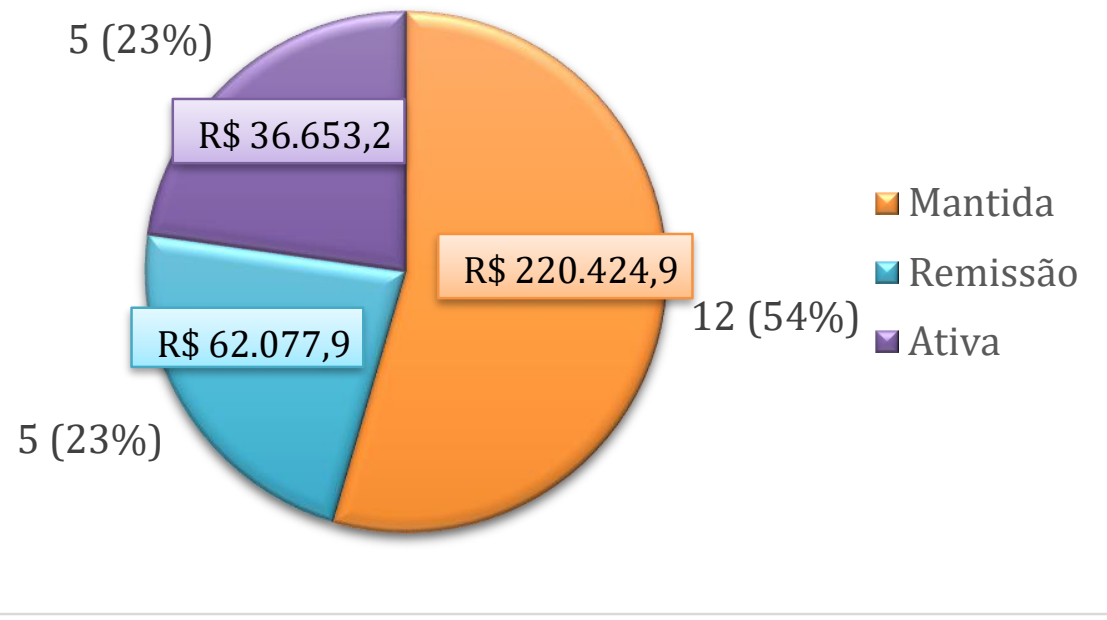

Dos pacientes que alcançaram um desfecho clínico favorável com CDAl $<220$ (5 pacientes), apenas 3 pacientes fizeram uso de biológicos anti TNF (seja de forma isolada ou combinada): 2 pacientes utilizaram ADA (em um total de 8 pacientes que fizeram uso deste fármaco) e 1 paciente utilizou INF (em um total de 7 pacientes que fizeram uso deste fármaco).

Tabela 6: Relações analisadas entre os medicamentos.

\begin{tabular}{|l|c|c|}
\hline & Adalimumabe & Infliximabe \\
\hline $\begin{array}{l}\text { Porcentagem de pacientes } \\
\text { que alcançaram ou } \\
\text { mantiveram desfecho } \\
\text { favorável dentre todos que } \\
\text { utilizaram tal medicação: }\end{array}$ & $7 / 8(87,5 \%)$ & $4 / 7(57,1 \%)$ \\
\hline $\begin{array}{l}\text { Custo Direto do tratamento } \\
\text { (para os pacientes que } \\
\text { alcançaram ou } \\
\text { mantiveram): }\end{array}$ & R\$ 208.929 & R\$ 68.828 \\
\hline $\begin{array}{l}\text { Taxa média de remissão } \\
\text { (em meses): }\end{array}$ & 41 meses/7 pacientes $=$ & 27 meses/4 pacientes = \\
\end{tabular}


Assim, demonstrou-se que pacientes em uso de Adalimumabe apresentaram taxa média de remissão equivalente a 5,85 meses enquanto os pacientes em uso de Infliximabe apresentaram equivalente a 6,75 meses. Desta forma, o Infliximabe apresentou taxa 1,153 vezes maior que a do Adalimumabe e, contudo, o custo direto do Adalimumabe baseado no preço de mercado foi superior em aproximadamente 3 vezes.

Meta-analise e revisão sistemática realizada recentemente demonstra que Infliximabe e Adalimumabe são considerados primeira-linha para indução de remissão em pacientes com DC moderada a severa. Sendo a Azatioprina primeira escolha para DC leve a moderada. (SINGH et al., 2018). Estudos apontam que o Infliximabe possui a maior probabilidade de ser efetivo como agente indutor de remissão (86\%), em contrapartida o Adalimumabe possui maior efetividade como terapia de manutenção de remissão (48\%). (STIDHAM et al., 2014). O que foi confirmado em nosso estudo, onde o Adalimumabe apresentou maior proporção de pacientes que alcançaram desfecho favorável dentre todos que utilizaram tal medicação, como o exposto na Tabela 6.

O objetivo principal do tratamento clínico com medicações é promover maior qualidade de vida do paciente e assim como manter paciente em remissão minimizando a necessidade de serem submetidos a procedimentos cirúrgicos. Portanto, foi analisado quantos pacientes tiveram recaídas com doença ativa e evoluíram com complicações necessitando de cirurgia. Nesta coorte, 20,83\% necessitaram de cirurgia. Dentre os pacientes que passaram por procedimento cirúrgico, cerca de $40 \%$ utilizam terapia biológica após cirurgia como tratamento de manutenção. Baseado nesse dado, pode-se concluir que o objetivo do tratamento clínico não foi alcançado, independente se com terapia biológica ou não. Dessa forma, ocasionando maiores despesas e prejuízos ao paciente e onerando o serviço público de saúde.

De acordo com Evaluation of health economics in inflammatory bowel disease (2017), cumulativamente, os sintomas e as complicações da doença aumentam o custo, tanto para o paciente quanto para o sistema de saúde, devido a relação com a provisão de cuidados e perda de produtividade econômica em jovens/adultos jovens, que requerem uma terapia a longo prazo, sem cura disponível.

Além disso, o tratamento possui mecanismo de ação envolvendo o sistema imunológico e suas respostas, ocorrendo efeitos adversos relacionados e infecções 
específicas consequentes, onde com frequência os pacientes requerem hospitalizações ou manejos cirúrgicos, que também aumentam o custo da terapêutica.

Analisando-se a coorte dos 22 pacientes em estudo, pormenorizada paciente a paciente na Tabela 5 evidenciada, correlacionada às informações referentes de custo direto do tratamento de manutenção para alcançar desfecho clínico com base no CDAI, no Gráfico 3: observou-se que o custo direto do tratamento de manutenção foi maior proporcionalmente ao tempo de terapia visando alcançar o desfecho clínico favorável com CDAI $<220$.

Além disso, evidenciou-se a partir dos resultados obtidos na tabela 5 que pacientes que utilizaram terapia combinada, as quais no estudo foram ADA + AZA ou INF + AZA, não necessariamente apresentaram maiores custos diretos relacionados ao tratamento de manutenção sem apresentarem correlação concreta com desfecho clínico favorável ao final do período de análise.

\section{CONSIDERAÇÕES FINAIS:}

De acordo com os resultados apresentados pelo presente estudo, é possível concluir que o RCUI foi mais frequente que a DC, que foi significativamente mais encontrado em mulheres jovens. Neste estudo, a proporção de pessoas de etnia parda foi de $50 \%$ da população estudada, com apenas $8 \%$ em negros. O fenótipo Montreal foi principalmente L3 (Ileocolônico) com comportamento predominantemente inflamatório. Os medicamentos anti-TNF foram prescritos em quase metade da amostra. Sendo que $20,83 \%$ dos pacientes necessitaram de cirurgia. Manifestações extraintestinais foram apresentadas em $61 \%$, principalmente no sistema musculoesquelético.

Desta forma, o Infliximabe apresentou taxa média de remissão 1,153 vezes maior que a do Adalimumabe, apesar do custo direto do Adalimumabe baseado no preço de mercado ter sido aproximadamente 3 vezes superior ao do Infliximabe. Na amostra $23 \%$ dos pacientes não alcançaram taxa de remissão.

À medida que novas drogas biológicas concorrentes continuam a chegar no mercado, faz-se necessário o desenvolvimento de estudos com enfoque em análises farmacoeconômicas adequadas em pacientes apropriados, visto a necessidade de auxílio no processo de tomada de decisão sobre a alocação de recursos em cuidados de saúde. 


\section{REFERÊNCIAS BIBLIOGRÁFICAS:}

1- Bechara CS et al; Caracterização de pacientes operados por doença de Crohn pela classificação de Montreal e identificação de fatores preditores de sua recorrência cirúrgica Rev. Col. Bras. Cir. 2015; 42(2): 097-105.

2- BEST, W. R. et al. Development of a Crohn's disease activity index. National Cooperative Crohn's Disease Study. Gastroenterology, [s. I.], 1976.

3- Boukercha A M-AH, Bouzidi A, Saoula $H$, Nakkemouche $M$, Roy $M$, Hugot JP, Touil Boukoffa C. NOD2 /CARD15 gene mutations in North Algerian patients with inflammatory bowel disease. World J Gastroenterol. 2015;21:7786-94.

4- BRASIL. Banco de Preços em Saúde (BPS) do Ministério da Saúde. Disponível em www.bps.saude.gov.br. Acesso em junho de 2018.

5- CLASSIFICAÇÃO DE VIENA PARA, Da et al. From the Vienna Classification to the New Montreal Classification: Phenotype Characterization and Clinical Evolution of Crohn's Disease. GE -J Port Gastrenterol, [s. I.], v. 18, p. 15-21, 2011.

6- Clayburgh DR SL, Turner JR. A porous defense: the leaky epithelial barrier in intestinal disease. Lab Invest. 2004;84:282-91.

7- ESTRAT, Insumos. Ministério da Saúde - Brasil. Certolizumabe pegol para o tratamento da doença de Crohn moderada a grave. Relatório de Recomendação n²39 Janeiro/2017. Conitec, [s. I.], p. 43, 2017.

8- GOMOLLÓN, Fernando et al. 3rd European evidence-based consensus on the diagnosis and management of Crohn's disease 2016: Part 1: Diagnosis and medical management. Journal of Crohn's and Colitis, [s. I.], v. 11, n. 1, p. 3-25, 2017.

9- Hugot JP CM, Zouali H, Lesage S, Cezard JP, Belaiche J, Almer S, Tysk C, O'Morain CA, Gassull M, Binder V, Finkel Y, Cortot A, Modigliani R, Laurent-Puig P, Gower-Rousseau C, Macry J, Colombel JF, Sahbatou M, Thomas G. Association of NOD2 leucine-rich repeat variants with susceptibility to Crohn's disease. Nature. 2001;31(411):599-603.

10- Hanauer SB FB, Lichtenstein GR, Mayer LF, Schreiber S, Colombel JF, Rachmilewitz D, Wolf DC, Olson A, Bao W, Rutgeerts P. Maintenance infliximab for Crohn's disease: the ACCENT I randomised trial. Lancet. 2002;359(9317):1541-9.

11- Inohara N OY, Fontalba A, Gutierrez O, Pons F, Crespo J, Fukase K, Inamura S, Kusumoto 
S, Hashimoto M, Foster SJ, Moran AP, Fernandez-Luna JL, Nunez G. Host recognition of bacterial muramyl dipeptide mediated through NOD2. Implications for Crohn's disease. J Biol Chem. 2003;278:5509-12.

12- Groschwitz KR H, SP. Intestinal barrier function: Molecular regulation and disease pathogenesis. J Allergy Clin Immunol. 2009;124:3-20.

13- KHANNA, Reena; FEAGAN, Brian G. Evaluation of Health Economics in Inflammatory Bowel Disease BT - Crohn's Disease and Ulcerative Colitis: From Epidemiology and Immunobiology to a Rational Diagnostic and Therapeutic Approach. In: BAUMGART, Daniel C. (Ed.). Cham: Springer International Publishing, 2017. p. 291-297.

14- LEE, H. J. et al.; Ophthalmologic manifestations in patients with inflammatory bowel disease - Intest Res 2017;15(3):380-387.

15- Molodecky NA SI, Rabi DM, Ghali WA, Ferris M, Chernoff G, Benchimol El, Panaccione R, Ghosh S, Barkema HW, Kaplan GG. Increasing incidence and prevalence of the inflammatory bowel diseases with time, based on systematic review. Gastroenterology. 2012;142(1):4654.

16- Nielsen $\mathrm{OH}$ A. Tumor Necrosis Factor Inhibitors for Inflammatory Bowel Disease. New England Journal of Medicine. 2013;369:754-62.

17- Pierre-Olivier Blotière JrmR, Amélie Barré , Antoine Racine, Alain Weill , Laurent PeyrinBiroulet, Franck Carbonnel , Francois Alla. Conditions of prescription of anti-TNF agents in newly treated patients with inflammatory bowel disease in France (2011-2013). Digestive and Liver Disease. 2016;48:620-5.

18- Singh S HH, Sangaralingham LR, Schilz SR, Kappelman MD, Shah ND, Loftus EV Jr. Comparative Effectiveness and Safety of Anti-Tumor Necrosis Factor Agents in Biologic-Naive Patients with Crohn's Disease. Clinical Gastroenterology and Hepatology 2016;16:1542-3565. 19- Rutgeerts P VS, Van Assche G. Biological Therapies for Inflammatory Bowel Diseases. Gastroenterology. 2009;136:1182-97.

20- Siew C., NG et al. Worldwide incidence and prevalence of inflammatory bowel disease in the 21st century: a systematic review of population-based studies. The Lancet, [s. I.], v. 390, n. 10114, p. 2769-2778, 2017.

21- SILVERBERG, Mark S. et al. Toward an integrated clinical, molecular and serological classification of inflammatory bowel disease: report of a Working Party of the 2005 Montreal World Congress of Gastroenterology. Canadian journal of gastroenterology = Journal 
canadien de gastroenterologie, [s. I.], v. 19 Suppl A, n. Suppl A, p. 5A-36A, 2005.

22- SINGH, S. et al. Systematic review and network meta-analysis: first- and second-line biologic therapies for moderate-severe Crohn's disease. Alimentary Pharmacology \& Therapeutics, [s. I.], v. 48, n. 4, p. 394-409, 2018.

23- STARK, Renee; KÖNIG, Hans-Helmut; LEIDL, Reiner. Costs of inflammatory bowel disease in Germany. PharmacoEconomics, [s. I.], v. 24, n. 8, p. 797-814, 2006.

24- STIDHAM, R. W. et al. Systematic review with network meta-analysis: the efficacy of antiTNF agents for the treatment of Crohn's disease. Alimentary Pharmacology \& Therapeutics, [s. I.], v. 39, n. 12, p. 1349-1362, 2014.

25- Targan SR HS, Van Deventer SJH, Mayer L, Present DH, Braakman T, DeWoody KL, Schaible TF, Rutgeerts PJ,. A Short-Term Study of Chimeric Monoclonal Antibody cA2 to Tumor Necrosis Factor $\alpha$ for Crohn's Disease. New England Journal of Medicine. 1997;337:1029-36.

26- Tracey D KL, Sasso EH, Salfeld JG , Tak PP. Tumor Necrosis fator antagonista mechanisms of action: A comprehensive review. Pharmacology \& Therapeutics. 2007;117:244-79.

27- Takeshima F, Yoshikawa D, Higashi S, Morisaki T, Oda H, Ikeda M. Machida H, Matsushima K, Minami H, Akazawa Y, Yamaguchi N, Ohnita K, Isomoto H, Ueno M, Nakao K. Clinical efficacy of adalimumab in Crohn's disease: a real practice observational study in Japan. BMC Gastroenterology (2016) 16:82.

28- Tracey D KL, Sasso EH, Salfeld JG , Tak PP. Tumor Necrosis fator antagonista mechanisms of action: A comprehensive review. Pharmacology \& Therapeutics. 2007;117:244-79. 